Case report
Department of

Dermatology,

Venereology and

Leprology,

Postgraduate Institute

of Medical Education

and Research,

Chandigarh, India

S Gupta

B Kumar

Correspondence to: Bhushan Kumar,

Department of Dermatology, Venereology and Leprology, PGIMER, Chandigarh-160 012 , India

kumarbhushan@hotmail.com

Accepted for publication 25 February 2000

\title{
Dorsal perforation of prepuce: a common end point of severe ulcerative genital diseases?
}

\author{
Somesh Gupta, Bhushan Kumar
}

Severe ulcerative genital diseases can cause destruction of the prepuce, glans, or sometimes of the whole penis (phagedena). We observed a characteristic pattern of partial destruction of the prepuce as a result of a wide variety of ulcerative genital diseases. Five patients, two with severe genital herpes, one with hidradenitis suppurativa, and two with donovanosis presented with perforation on the dorsal surface of the prepuce. In four of them, the glans protruded through the defect and in one, the defect was not large enough to allow protrusion of the glans. In two patients, the preputial sac was obliterated. The relatively decreased blood supply of the prepuce is the probable explanation for perforation at this selective site.

(Sex Transm Inf 2000;76:210-212)

Keywords: prepuce; genital ulcer

\section{Introduction}

Genital ulcers causing rapid destruction of the glans and the prepuce or sometimes of the whole penis (phagedena) have been known since antiquity. ${ }^{1}$ Both sexually and nonsexually transmitted infections have been found to be associated with such a severe destructive process. ${ }^{1}$ We observed a characteristic pattern of the partial destruction of the dorsal prepuce, leading to perforation due to entirely unrelated aetiologies. Five such cases are reported here.

\section{Case reports}

CASE 1

A 31 year old man, a known HIV positive patient, presented with severe painful genital ulcers. Examination revealed large, well defined erosions with irregular margins, distributed over the glans, prepuce, and shaft of the penis. The inguinal lymph nodes were enlarged and tender. A diagnosis of genital herpes was made after exclusion of other STDs by relevant investigations and the patient was started on oral aciclovir at a dose of $400 \mathrm{mg}$ five times a day. The patient defaulted on follow up and drug compliance could not be confirmed. On examination, the prepuce had perforated from the dorsal surface and the glans protruded through it. The remaining part of the preputial sac healed with fibrosis leading to the obliteration of the sac.

CASE 2

A 28 year old man, a diagnosed case of hidradenitis suppurativa, presented with abscesses and discharging sinuses in both axillae, right arm, groin, and scrotum associated with moderate fever. He also had one discharging sinus over the dorsum of the prepuce. Examination revealed maceration in the whole inguinal region with foul smelling seropurulent discharge. A false passage in the preputial sac was present from which the glans was protruding.

The patient was more worried about his basic disease and was not much concerned about the deformity of the penis. No extra treatment was given other than that for hidradenitis suppurativa.

CASE 3

A 26 year old man presented with preputial perforation on its dorsal aspect, through which the glans was protruding (fig 1). The patient gave history of an ulcer on the undersurface of the dorsal prepuce of about $1 \frac{1 / 2}{2}$ months' duration, which was mildly painful and bled easily on touch. He received some antibacterial agents but without much relief. The dorsal aspect of the prepuce was ultimately eaten up by the ulcer through which the glans

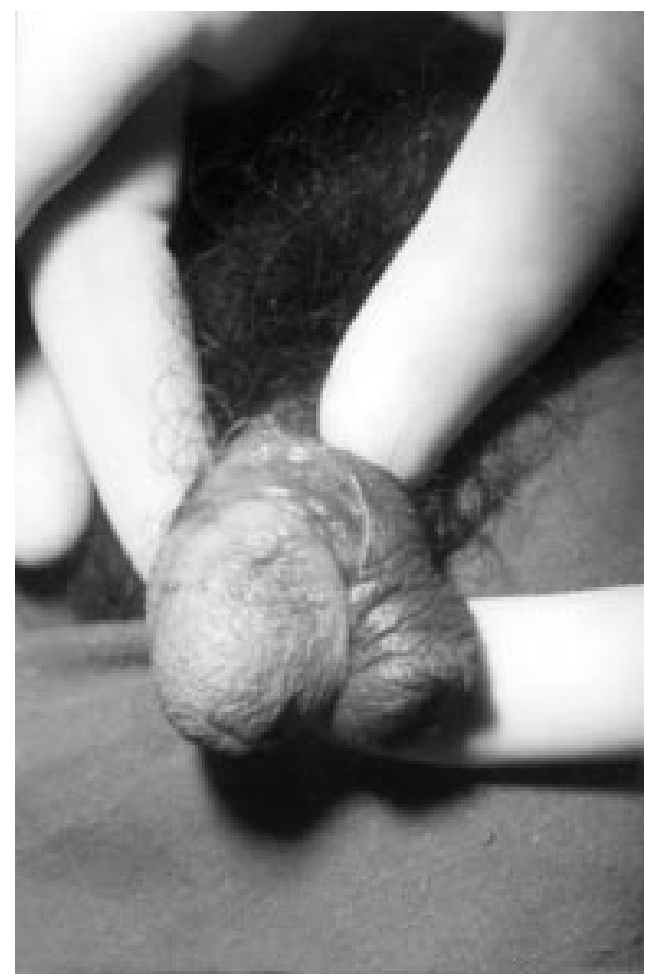

Figure 1 Dorsal preputial perforation, possibly due to donovanosis. 
protruded. An ulcer with healthy granulation tissue was present over the prepuce and the adjacent exposed part of the coronal sulcus. The clinical picture was suggestive of donovanosis. The tissue smear for Donovan bodies was negative and all the other STIs were excluded by the appropriate tests. The patient was empirically treated with doxycycline for 3 weeks with the diagnosis of donovanosis. His lesion healed but the deformity persisted.

CASE 4

A 29 year old man presented with two ulcers on the penis, which had appeared around 2 months earlier. The treatment he took failed to heal the lesions and one of them eroded a part of the foreskin. Examination revealed a swollen penis with a circular hole on the dorsal aspect of the prepuce through which an ulcer on the glans was visible. The ulcer had raised margins with central depression. Another ulcer was present on the intact preputial skin on the ventrolateral aspect. This ulcer had exuberant granulation tissue and a well defined margin. A clinical diagnosis of donovanosis was made which was confirmed by demonstration of the Donovan bodies in the tissue smear. Other investigations including those for syphilis, chancroid, and HIV were negative. The patient was treated with doxycycline $100 \mathrm{mg}$ twice daily for 6 weeks. The ulcers healed, though the circular defect in the prepuce persisted. The patient was not much concerned about the anatomical defect in the prepuce.

CASE 5

A 24 year old man, a known HIV positive patient, presented with a fibrosed foreskin. $\mathrm{He}$ gave a history of recurrent ulcerations on the prepuce and the glans for past 2 years which healed each time on treatment with aciclovir. The patient was unable to explain the deformity at the time of presentation. Examination revealed a fibrosed and obliterated prepuce positioned on the ventral aspect. The glans protruded through a large defect on the dorsal aspect. All relevant investigations were not contributory. A diagnosis of recurrent genital herpes was made considering the history and response to the aciclovir therapy.

Cases 1 and 5 were HIV antibody positive. All patients with STIs were heterosexual and did at times indulge in the unsafe sexual practices. All were advised circumcision. All were not much concerned about their anatomical deformities.

Table 1 Summary of the reported and present cases of dorsal perforation of prepuce

\begin{tabular}{|c|c|c|c|c|c|}
\hline No & $\begin{array}{l}\text { Age } \\
\text { (years) }\end{array}$ & $\begin{array}{l}\text { Suspected cause of } \\
\text { perforation }\end{array}$ & $\begin{array}{l}\text { Protrusion of } \\
\text { glans through } \\
\text { the defect }\end{array}$ & $\begin{array}{l}\text { Obliteration of } \\
\text { preputial sac }\end{array}$ & Reference \\
\hline 1 & 24 & $\begin{array}{l}\text { ?Warts } \\
\text { ?Podophyllin }\end{array}$ & No & No & 2 \\
\hline 2 & 27 & $\begin{array}{l}\text { ?Donovanosis } \\
\text { ?Chancroid }\end{array}$ & Yes & No & 3 \\
\hline 3 & 35 & Unknown & No & No & 4 \\
\hline 4 & 31 & Genital herpes & Yes & Yes & Present report \\
\hline 5 & 28 & Hidradenitis suppurativa & Yes & No & Present report \\
\hline 6 & 26 & ?Donovanosis & Yes & No & Present report \\
\hline 7 & 29 & Donovanosis & No & No & Present report \\
\hline 8 & 24 & Genital herpes & Yes & Yes & Present report \\
\hline
\end{tabular}

\section{Discussion}

A literature search revealed that only three cases with perforation of the prepuce have been reported..$^{2-4}$ Like our cases, the site of perforation in these cases was the dorsal surface of the prepuce (table 1). In one of these reported cases the cause of the perforation was delayed podophyllin reaction. ${ }^{2}$ The dorsal perforation in the second reported patient was correlated with chancroid or donovanosis, though the diagnosis was not confirmed. ${ }^{3}$ In the third reported patient, the cause was unknown; however, some trauma in childhood was suspected. ${ }^{4}$ Relatively increased susceptibility of dorsal prepuce to perforation is further strengthened by the finding of only the dorsal perforation in our first, fourth, and fifth patients in spite of the presence of the ulcer(s) on the other aspects of the prepuce. We were fascinated with this characteristic presentation and attempted to find an explanation.

The prepuce is a penta laminar specialised junctional tissue with mucosa on its inner surface and skin on its outer surface. ${ }^{5}$ There has been an inconclusive debate on its value in sexual intercourse and in preventing sexually transmitted diseases. ${ }^{67}$ The preputial blood supply originates from its proximal attachment with the skin of penile shaft. ${ }^{8}$ The prepuce is not supplied by vessels distally from the corona. ${ }^{8}$ The superficial penile arteries supplying the prepuce on reaching the preputial ring become branched, tortuous, and minute. ${ }^{9}$ However, the ventral aspect of the preputial skin adjacent to the frenulum is richly supplied by a separate artery - that is, frenular branch of the dorsal artery of the penis. ${ }^{8} 9$ Thus, the vascular supply of the dorsal prepuce is relatively deficient in comparison with its ventral aspect and the skin over the shaft. It is well known that structures richly supplied by blood vessels are less vulnerable to infection and more able to combat it once it occurs. Severe destructive and necrotic processes at any site usually occur owing to the inflammation produced by the infection causing an interruption in the blood supply to the area. ${ }^{10}$ Thus, the areas with relatively less blood supply are more prone to this type of ischaemic necrosis. The ulcerations by non-infectious causes are also likely to cause severe necrosis in the areas less profusely supplied by blood vessels. This may be a possible explanation for the perforation of the same site of prepuce owing to different genital diseases in our patients. We presume that the genital ulcers in our patients began on the less vascular inner (mucosal) surface and eroded through the outer (skin) surface.

One very interesting fact in ours as well as other two reported patients was their lack of concern about the very obvious deformity of an important part of the human anatomy. The other reported patient had discomfort during intercourse, which he ignored for years. ${ }^{4}$ Absence of pain and discomfort during the intercourse and concern about the more serious basic disease are the possible reasons for unconcern and recourse to surgery. The other two healthy patients probably were more happy to retain the prepuce. 
In conclusion, the dorsal perforation of the prepuce is a sequel of the genital ulcer diseases due to a wide variety of aetiologies. It is probably related to the vulnerability to necrosis of the part that has relatively less blood supply. Awareness of this presentation among genitourinary physicians and dermatologists should encourage more studies and reports.

Contributors: Both authors are engaged in the running of the STD clinic on the regular basis. The collection of the materials and the writing of the manuscript are undertaken collectively by both.

1 Oriel JD. Phagedena. Genitourin Med 1994;70:218-21.

2 Maite H, Hay KR. Mystery of the holey prepuce: delayed podphyllin skin damage? Genitourin Med 1989;65:201-7.
3 O'Farrell N. Holey prepuce following genital ulceration. Genitourin Med 1990;66:48-9.

4 Agrawal V, Raghavan L, Dargan P. A unique cause of male dyspareunia. F Indian Med Assoc 1999;12:526.

5 Cold CJ, Taylor JR. The prepuce. Br f Urol Int 1999; 83(suppl 1):34-44.

6 Fleiss PM, Hodges FM, Van Howe RS. Immunological functions of the human prepuce. Sex Transm Inf 1998;74: 364-7.

7 Moses S, Bailey RC, Ronald AR. Male circumcision: assessment of health benefits and risks. Sex Transm Inf 1998;74:368-73.

8 Hinman F Jr. The blood supply to preputial island flap. $\mathcal{f}$ Urol 1991;145:1232-5.

9 Hinman F Jr. Penis and male urethra. In: Atlas of urosurgical anatomy. Philadelphia: WB Saunders, 1993:41845.

10 Jackson R, Bell M. Phagedena: gangrenous and necrotic ulcerations of skin and subcutaneous tissue. Can Med Assoc f 1982;126:363-8. 